\title{
Characterization of flagellins isolated from a highly motile strain of Lactobacillus agilis
}

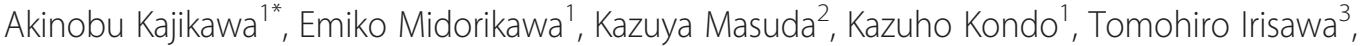 \\ Shizunobu Igimi ${ }^{2}$ and Sanae Okada ${ }^{1}$
}

\begin{abstract}
Background: Most lactic acid bacteria are non-motile but some of them are flagellated and exhibit motility. So far, motile lactobacilli have rarely been studied, and characteristics of their flagellins are poorly understood. In this study, a highly motile strain of Lactobacillus agilis was recruited for transcriptional analysis and characterization of its flagellins.

Results: Unlike another motile lactic acid bacteria of intestinal isolate, Lactobacillus ruminis, flagellar filaments of the L. agilis strain probably consist of two homologous but distinct flagellins. Glycosylation of the flagellar filaments and their resistance to heat, acid and SDS were also observed. The immunological activity of the flagellins was evaluated through the stimulation of Caco-2 cells. The results show that TLR5-stimulating activity of the protein is attenuated, likely due to an incomplete TLR5-recognition site.
\end{abstract}

Conclusions: The flagella filaments of L. agilis BKN88 consist of two homologous glycosylated flagellins, which likely have an incomplete TLR5-recognition site. The characteristics of the flagellin are presumably a consequence of adaptation as a commensal microbe in the gastrointestinal tract.

\section{Background}

While lactic acid bacteria are generally non-motile, some of them are flagellated and exhibit motility. Lactobacillus agilis and Lactobacillus ruminis are representatives of those flagellated microbes which have been primarily isolated from homeothermal animals such as cows, pigs, birds, etc. [1-5]. So far, a few studies on these lactobacilli have been done, but their characteristics are barely understood. Flagella have a complex protein structure and their filaments are made up of a subunit protein referred to as flagellin. Flagellins are also known as ligands of Toll-like receptor 5 (TLR5), one of pattern recognition receptors [6]. In a previous study, Neville et al. presented the genetic and transcriptional analysis of the motility-associated genes of L. ruminis. The immunological activity of flagellin isolated from $L$. ruminis and a few other flagellated lactobacilli via TLR5 was also reported, although flagellins of $L$. agilis were not included [7].

Our group has recruited $L$. agilis as a model of motile lactobacilli which reside in the gastrointestinal mucosa.

\footnotetext{
*Correspondence: a3kajika@nodai.ac.jp

'Department of Applied Biology and Chemistry, Tokyo University of

Agriculture, 1-1-1 Sakuragaoka, Setagaya, Tokyo 156-8502, Japan

Full list of author information is available at the end of the article
}

Although $L$. agilis is frequently isolated from birds, there are other isolates from pigs and humans [8, 9]. In addition, we isolated a few $L$. agilis strains from lemurs and tapirs (unpublished). Hence, $L$ agilis may be considered as a commensal distributed among various animals. A major advantage in the study of this species is that it is capable of transformation with well-established plasmid vectors such as pGK12 and derivatives [10] (our unpublished data). Thus $L$ agilis can be investigated using genetic manipulations or applied as biotherapeutics, such as vaccine/ drug-delivery systems [11]. While L. agilis appears to be a promising model, however, it has not been characterized sufficiently.

This study focuses on the flagellins of a highly motile strain, L. agilis BKN88, which was derived from a chicken isolate strain, JCM 1048. Firstly, the expression profile of the two copies of flagellin-encoding genes was determined. Secondly, posttranscriptional modification of the proteins was shown, and the stability of the flagellar filaments under depolymerizing conditions was tested. Lastly, the immunological activity of the flagellins was compared to that of the orthologous proteins of pathogenic bacteria in their interaction with Caco-2 cells. The aim of this 
study is to reveal previously unknown characteristics of flagellins of the motile commensal $L$. agilis.

\section{Results}

\section{Motility of L. agilis strains}

The motility of each L. agilis strain was confirmed in MRS soft-agar culture. JCM 1048 and JCM 1049 spread in the semi-solid medium while JCM $1187^{\mathrm{T}}$ and L. paracasei IGM393 did not (Fig. 1a). The motile strains were then observed by microscopy; however, only a small fraction of the cells were actively swimming. In order to isolate a uniformly motile cell culture, hundreds of subcultures of JCM 1048 and JCM 1049 were investigated. With this screening, a highly motile subculture of JCM 1048, identified as BKN88, was obtained. In the culture of BKN88, almost all cells were actively moving at exponential phase, with decreasing motility at late exponential phase to stationary phase. TEM analysis showed multiple filaments around the BKN88 cells, indicating peritrichous flagella of the bacterium.

\section{Expression of flagellin genes}

Based on the sequence of L. agilis DSM $20509^{\mathrm{T}}$, which has recently been deposited to GenBank, a partial gene map of the motility operon is shown in Fig. 2a. Two flagellin-encoding genes with $90 \%$ homology of both their nucleotides and amino acids are located in tandem. To discriminate the transcription of the two homologous flagellin-encoding genes, $f l i C 1$ and $f l i C 2$, specific primers were designed. Since only the amplicon of fliC1 includes a HindIII site, the specificity of the primers were easily validated by digestion of the PCR products with the restriction enzyme (Fig. 2b). RT-PCR to detect transcription of fliC1/fliC2 in BKN88 was performed at early exponential phase, when the culture showed the highest motility. As shown in Fig. 2c, expression of both $f l i C 1$ and $f l i C 2$ were detected.

\section{Composition and posttranslational modification of the flagellins}

Flagellar filaments of BKN88, L. monocytogenes EGD, and $S$. Typhimurium $92-35$ were isolated by ultracentrifugation. The isolated proteins were then analyzed by SDSPAGE and CBB staining. As shown in Fig. 3a, double bands which have slightly different molecular mass from each other were detected from BKN88. To identify these two proteins, each band was collected separately and analyzed by MALDI-TOF MS after digestion with trypsin. The mass spectra obtained from the two protein bands appeared to be similar, with minor differences (Fig. 4). Although the overall coverage of the peptide mass fingerprinting was low, two major peak of both mass spectra were consistent with those of peptides from FliC1 (the lower band) and FliC2 (the upper band). Since there are multiple glycosyltransrerase genes at flanking region of the flagellin genes (Fig. 2a), glycosylation of the flagellins was predicted. PAS-staining of acrylamide gels indicated that both $\mathrm{FliC} 1$ and $\mathrm{FliC} 2$ underwent posttranslational modification (Fig. 3b). Neither the flagellins of $S$. Typhimurium nor those of $L$. monocytogenes were stained with PAS as already known, albeit the flagellin of $L$. monocytogenes was glycosylated with N-acetylglucosamine [12].

\section{Depolymerization of flagellar filaments}

The stability of the flagellar filaments of L. agilis BKN88 and $S$. Typhimurium 92-35 were assessed by heating, and treatment with acid and detergent. After the purified flagellar filaments were incubated under the each condition, the proteins were applied to Native-PAGE gels. Since flagellar filaments are macromolecules, only the depolymerized proteins can travel in the gel. As shown in Fig. 5, the flagellar filaments of L. agilis BKN88 were more resistant to thermal or acidic conditions than those of $S$. Typhimurium 92-35. While the flagella of $S$. Typhimurium were depolymerized even at $37{ }^{\circ} \mathrm{C}$, those a

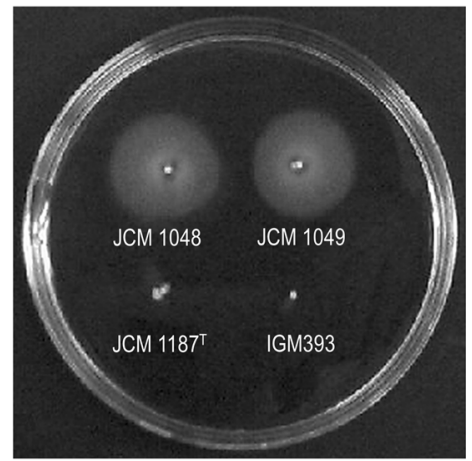

b

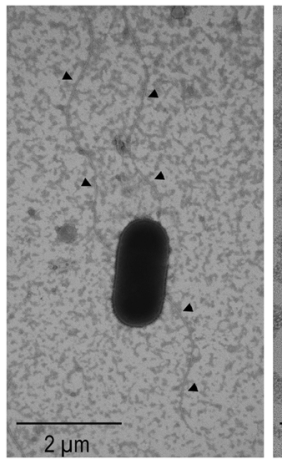

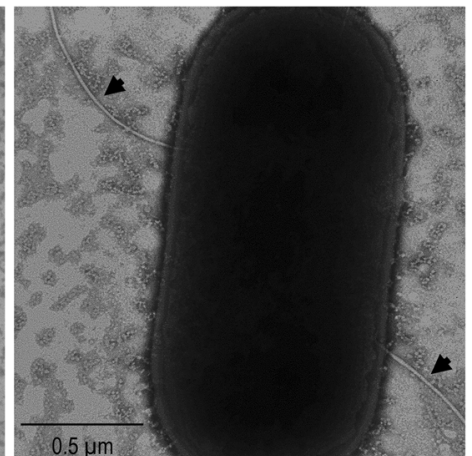

Fig. 1 Observation of motility and flagella. a The motility of L. agilis strains was determined using semi-solid MRS-agar medium. Three L. agilis strains and L. paracasei IGM393 (non-motile) were stabbed and incubated overnight. b TEM image of L. agilis BKN88 grown on an MRS-agar plate. The rates of magnification were 13900:1 (left) and 76600:1 (right). Arrow heads point flagellar filaments of BKN88 


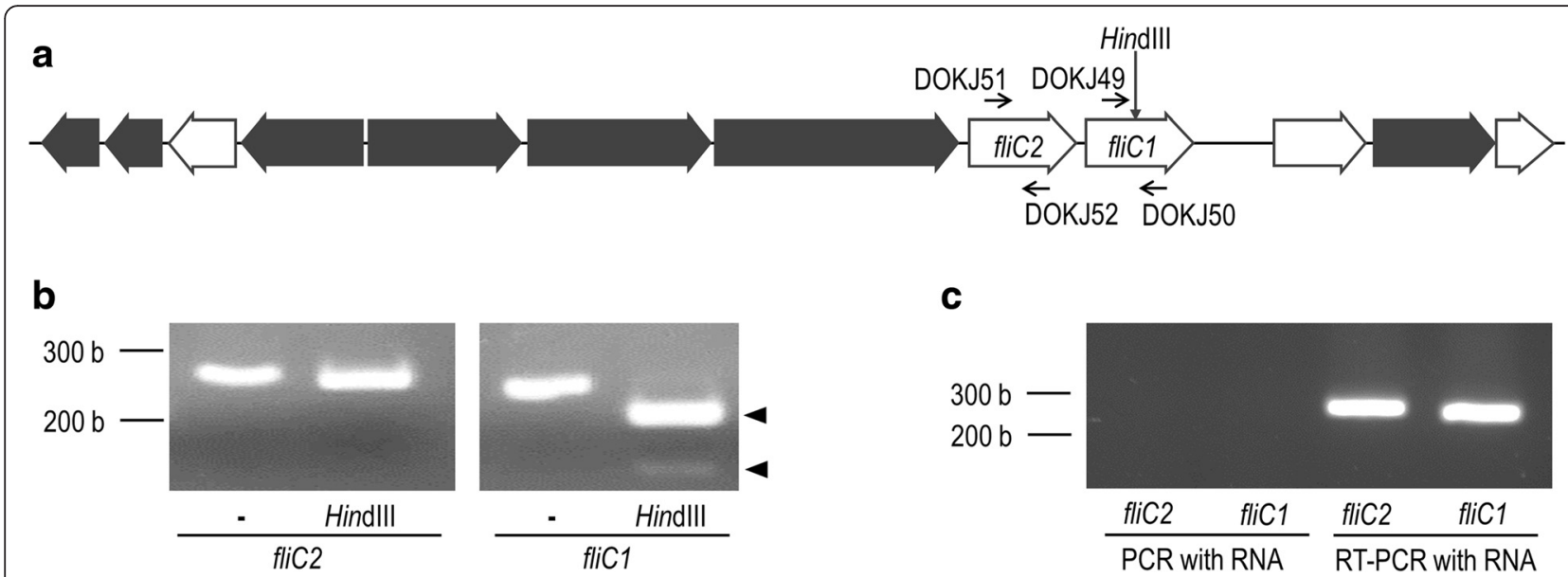

Fig. 2 Genetic analysis of the flagellin-encoding genes of L. agilis BKN88. a Partial gene map of the L. agilis motility operon. PCR amplification primers and the HindllI site are shown. Filled arrows represent putative glycosyltransferases. $\mathbf{b}$ Validation of flagellin-specific primers. Partial fragments of fliC1 and fliC2 were amplified by PCR using the specific primers and digested with Hindlll. c Transcription of fliC1/fliC2 was detected by RT-PCR. PCR without reverse transcription was also performed to detect contaminated DNA

of L. agilis were partially disassembled only at $57{ }^{\circ} \mathrm{C}$. Monomeric flagellins of $L$. agilis were detected after treatment with $5 \mathrm{mM} \mathrm{HCl}$; however, $0.5 \mathrm{mM} \mathrm{HCl}$ was sufficient to depolymerize the flagellar filaments of $S$. Typhimurium. Differences between the flagella of the two organisms were not observed under SDS-treatment.

\section{Immunological activity of the flagellins}

IL- 8 production from Caco- 2 cells induced by stimulation with flagella/flagellins was assessed. With native flagella (filaments) of $L$. agilis BKN88, no clear IL-8 production was detected, while high levels of IL- 8 was observed by stimulation with those of $S$. Typhimurium $92-35$ and $L$. monocytogenes EGD (Fig. 6a). The same assay was performed with depolymerized flagellins prepared by chaotropic denaturation. After this treatments, the IL-8 response was slightly increased in $L$. agilis flagellins, but still much lower than that induced by $S$. Typhimurium (Fig. 6b). The conserved amino acid residues of bacterial flagellins which are critical for TLR5-recognition of $L$. agilis and other bacteria were compared (Fig. 6c). Most of the amino acid residues were conserved in the lactobacilli; however, there were a few differences between the commensal bacteria and the TLR5-stimulating pathogens. While $S$. Typhimurium and L. monocytogenes have -LQRin the TLR5-recognition site, L. ruminis and L. agilis instead have -LGR- and -LNR- respectively. Moreover, two other differences at the site were found in L. agilis flagellins.

\section{Discussion}

Motile lactobacilli have rarely been studied, and as commensals, the significance of their flagella remains unknown. With increasing interest in the immunological
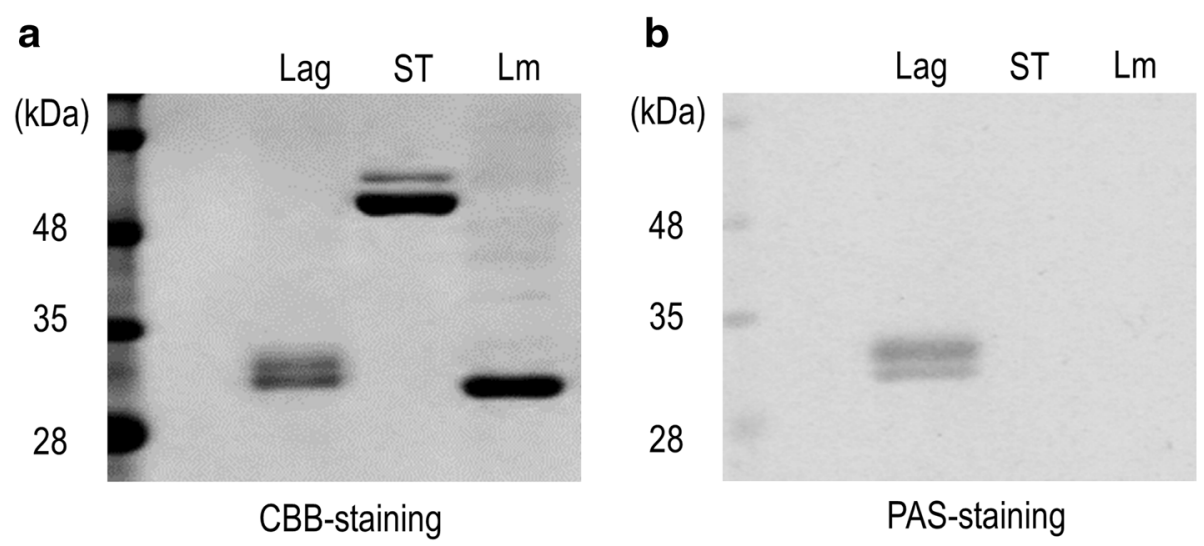

Fig. 3 SDS-PAGE and staining. Isolated flagellins from L. agilis BKN88 (Lag), S. Typhimurium 92-35 (ST), and L. monocytogenes EGD (Lm) were separated by SDS-PAGE and stained with CBB (a) or PAS (b). The molecular mass of the standard marker bands are shown at the left margin 


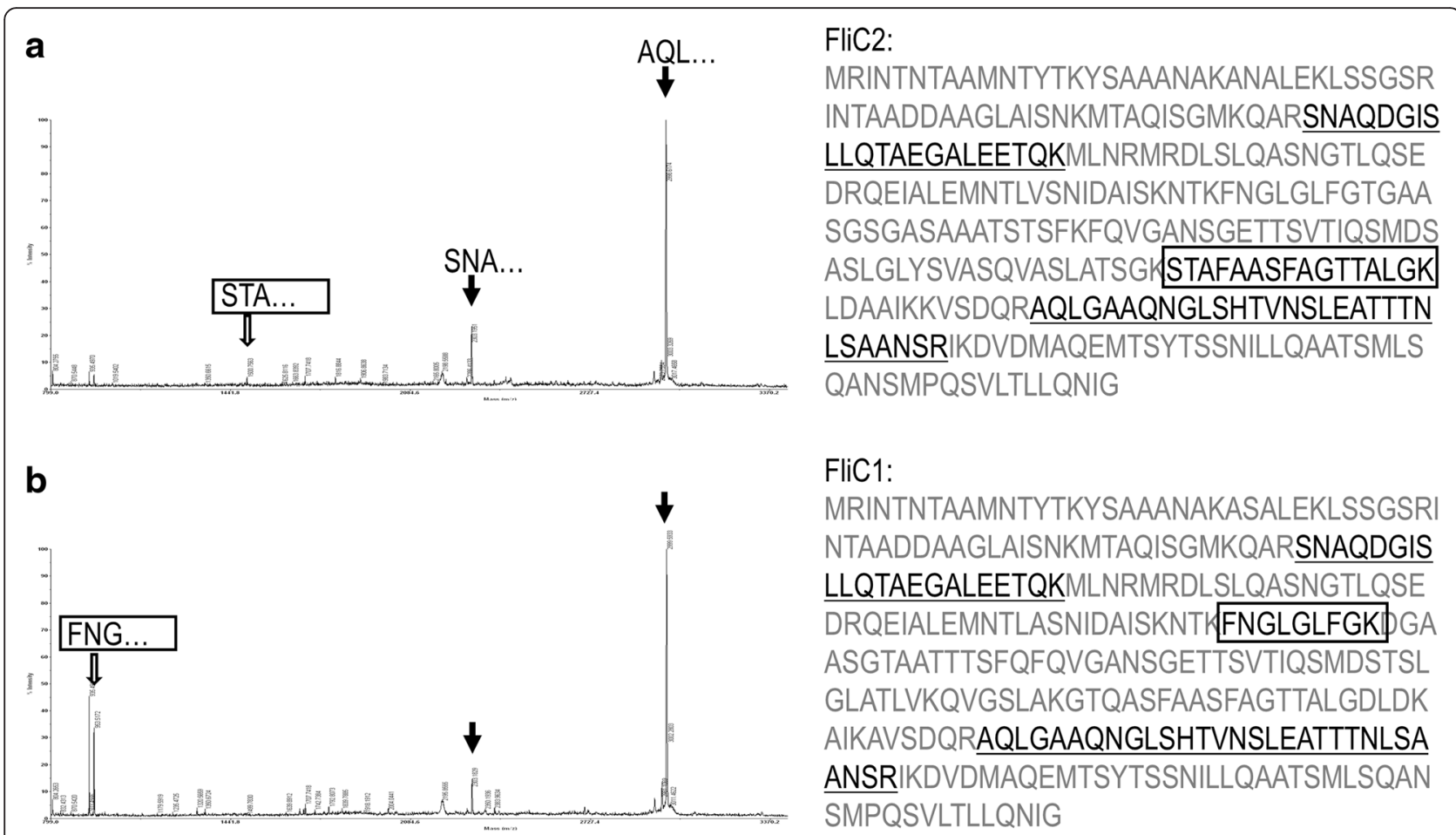

Fig. 4 Identification of flagellins by MALDI-TOF MS analysis. Protein bands were separately collected from SDS-PAGE gels and analyzed. Mass spectra of the upper band (a) and lower band (b) are shown. Filled arrows represent signals consistent with the mass of underlined peptides. Open arrows represent signals consistent with boxed peptides

properties of lactic acid bacteria, the unique ability of the flagella of L. agilis to interact with TLR5 is of particular note. In this study, flagellins of L. agilis BKN88 were analyzed and some of their unique characteristics have been uncovered.

The flagellar filaments of $L$. agilis BKN88 consist of two homologous proteins, FliC1 and FliC2. This was confirmed at both the mRNA and protein level, although the coverage of peptide mass fingerprinting was low, possibly due to glycosylation of the flagellins which affects the mass of peptides. Previously, Neville et al. reported that a motile strain of $L$. ruminis ATCC 27782, which also has two flagellin genes, expressed fliC2 dominantly and fliC1 only slightly [7]. These facts indicate that flagellin-gene expression and composition of flagellins in the filaments are not uniform among motile lactobacilli.

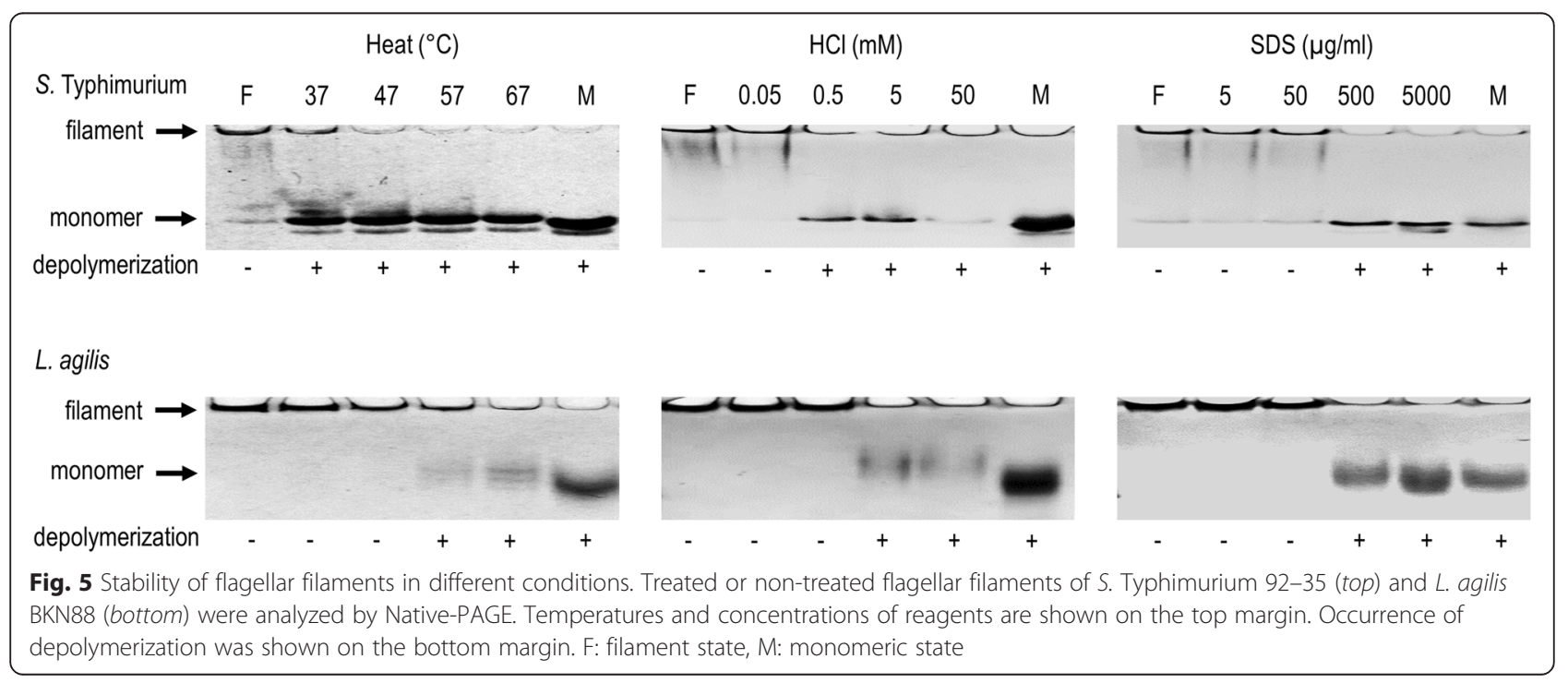




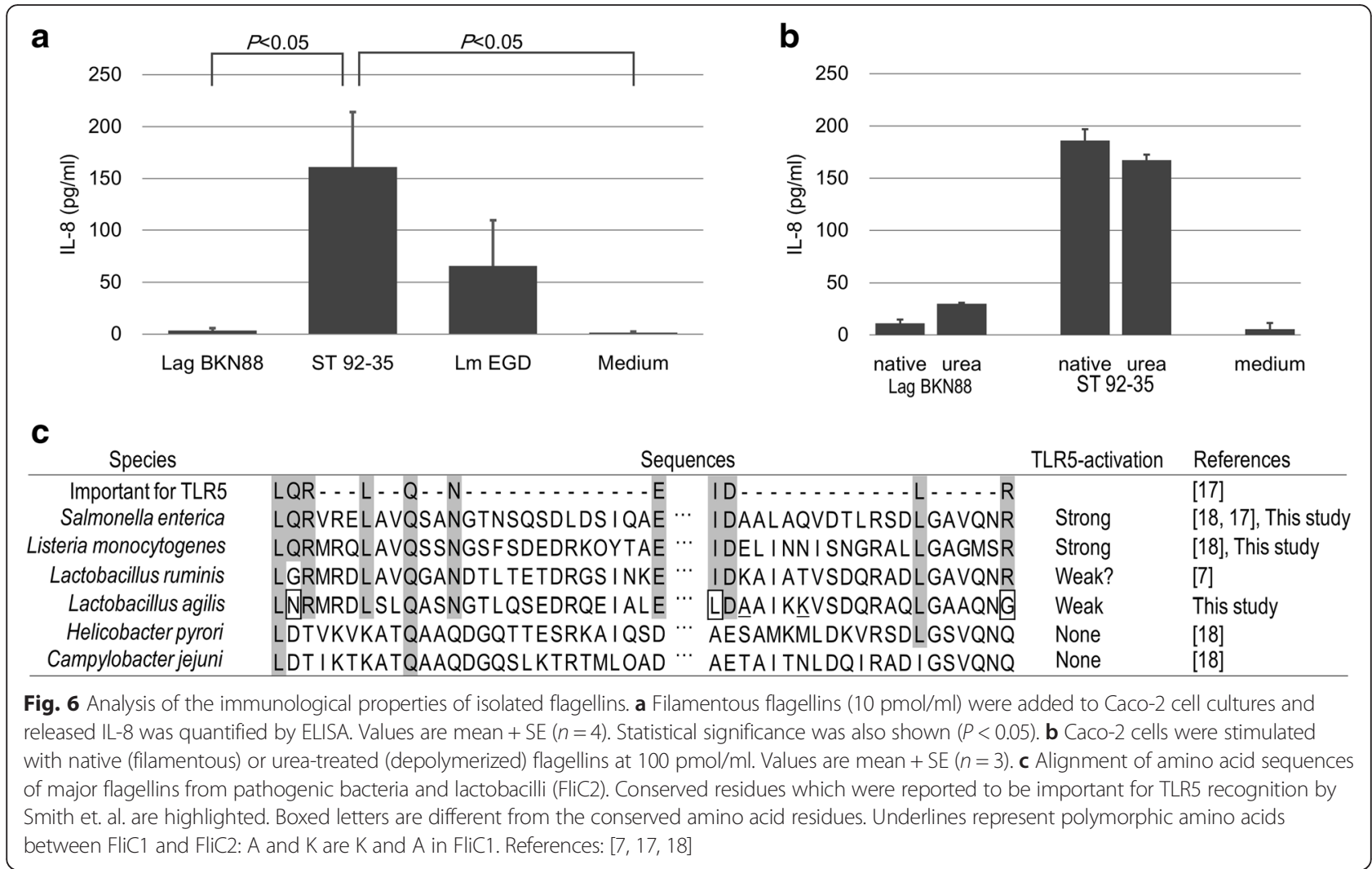

PAS-staining showed that both the $\mathrm{FliC} 1$ and $\mathrm{FliC} 2$ flagellins were glycosylated. This posttranscriptional modification was previously reported in other bacteria and archaea [13, 14]. In those studies, glycosylation of flagellins was reported to be involved in stability, assembly, virulence, or motility of flagella or the flagellated microbes, albeit the functions are not fully understood. Types of molecular bonds between a flagellin and a glycan are either N- or O-linkage. Since only O-linkage bonds are found in Gram-positive bacteria, it is likely that lactobacilli have the same type of bond. A previous study showed that glycosylated flagellins of Pseudomonas strains were more immunologically active than unmodified flagellins [15]. Hence, the glycosylation of Lactobacillus flagellins might have an impact on their immunological properties. Further studies need to be done to investigate mechanisms and influences of the modification.

The flagellar filaments of $L$. agilis were relatively stable under acidic conditions. This characteristic seems to be reasonable because lactobacilli are routinely producers of lactic acid. The flagellar polymeric structure is found to be thermostable compared to that of Salmonella. Collectively, the flagella of L. agilis may be durable, which would support sustainable motility of the microbe. Resistance to depolymerization also suggests that the flagella are immunologically inactive, since TLR5 interacts with only monomeric flagellin but not with polymeric states $[16,17]$.
Indeed, the native flagella of $L$. agilis barely induced IL-8 production by Caco-2 cells while those of $S$. Typhimurium elicited the response due to their depolymerization at $37{ }^{\circ} \mathrm{C}$. This might contribute to the symbiotic relationship between $L$. agilis and its host, by avoiding undesired immunostimulation.

As previously reported, the flagellins of $L$. ruminis present their immunological activity via interaction with TLR5 [7]. Likewise, monomeric flagellins of L. agilis exhibit the proinflammatory characteristic; however, the activity was found to be much lower than that of $S$. Typhimurium and L. monocytogenes in this study. Previously, specific amino acid residues conserved among the D1 domain of Salmonella FliC were proven to be critical for TLR5recognition [17]. The independent study by Verma et al. also demonstrated that replacement of amino acids at this conserved site of the Pseudomonas aeruginosa flagellin drastically reduced IL-8 response via TLR5 [15]. H. pylori and $C$. jejuni replaced most of those amino acid residues in their flagellins and thus lack TLR5-stimulating activity. Furthermore, a chimeric Salmonella FliC in which the TLR5-recognition site was replaced with the corresponding site of flagellin from H. pylori failed to interact with TLR5, which supports the importance of those amino acid residues [18]. In Lactobacillus flagellins, a few mismatchs in those sequences conserved among high TLR5activators such as the flagellins of $S$. Typhimurium and 
L. monocytogenes were found. Hence, it is likely that the attenuated immunological activity of $L$. agilis flagellins are attributed to the slight but influential differences at the TLR5-recognition site. As a symbiotic bacterium, $L$. agilis may not be involved in proinflammatory responses at the gastrointestinal mucosa. Presumably, the attenuation on the TLR5 ligand could help the bacterium to avoid exclusion by the local immune system of the hosts.

\section{Conclusions}

The flagella filaments of L. agilis BKN88 consist of two homologous glycosylated flagellins and are relatively stable in acidic and thermal conditions. The flagellins likely have an incomplete TLR5-recognition site, which may be the reason for their attenuated immunological activity. These characteristics of the flagellin are presumably a consequence of adaptation as a commensal microbe.

\section{Methods}

\section{Bacterial strains and growth conditions}

Lactobacillus paracasei IGM393 (former ATCC393, laboratory strain) [19], Lactobacillus agilis JCM 1048 (avian isolate), JCM 1049 (porcine isolate), JCM $1187^{\mathrm{T}}$ (swage water isolate), and BKN88, a highly motile variant of JCM 1048, were used in this study. All JCM strains were purchased from RIKEN BioResource Center, Ibaraki, Japan. L. agilis strains were grown statically (liquid culture) or anaerobically (plate culture) using AnaeroPouch-Anaero Anerobic Gas Generators (Mitsubishi Gas Chemical) in MRS broth/agar (BD) at $37{ }^{\circ} \mathrm{C}$. Motilities of lactobacillus strains were determined by inoculation into semi-solid MRS medium with $0.2 \%$ agar. Bacterial motility was also observed using an optical microscope. Salmonella enterica subsp. enterica serovar Typhimurium 92-35 (laboratory stock) was grown aerobically (shaking) in Brain Heart Infusion (BHI) broth (BD) at $37^{\circ} \mathrm{C}$. Listeria monocytogenes EGD [20] was grown statically in BHI broth at $25^{\circ} \mathrm{C}$.

\section{Transmission electron microscopy (TEM)}

Bacterial cells prepared from colonies on a MRS-agar plate were absorbed to formvar film coated copper grids (400 mesh) and stained with $2 \%$ phosphotungstic acid solution ( $\mathrm{pH}$ 7.0). The bacterial cells and the flagellar filaments with negative staining were visualized using a transmission electron microscope (JEM-1400Plus, JEOL Ltd., Tokyo, Japan) at an acceleration voltage of $80 \mathrm{kV}$. Digital images were taken with a CCD camera (VELETA, Olympus Soft Imaging Solutions $\mathrm{GmbH}$, Münster, Germany).
PCR and reverse transcriptional PCR (RT-PCR)

Specific primers for detection of each flagellin-encoding gene (fliC1 and $f l i C 2$ ) were designed in reference to the sequence of the $L$. agilis DSM $20509^{\mathrm{T}}$ motility operon (GenBank, Acession\# KM886859). Primer pairs, DOKJ49 (CTT TGG AAA AGA TGG TG) and DOKJ50 (CAG CCT TGA TAG CTT T) for flic1, DOKJ51 (TTT CGG TAC AGG TGC A) and DOKJ52 (CTT TCT TGA TAG CAG C) for $f l i C 2$, were used for PCR. Total RNA was prepared from bacterial cultures at early exponential phase. Fresh MRS broth was inoculated with a $1 \%$ volume of overnight culture of $L$. agilis BKN88 and incubated until the optical density $(600 \mathrm{~nm})$ reached 0.2 . Cells were collected and washed twice with TE buffer, followed by bead beating with a FastPrep Instrument (MP Biomedicals) in RNAprotect Bacteria Reagent (QIAGEN). The bacterial RNA was purified using an RNeasy Mini Kit (QIAGEN) in accordance with the manufacturer's instruction. RT-PCR was performed using a PrimeScript One Step RT-PCR kit (Takara). Contamination of DNA in the RNA sample was tested for using Ex. taq DNA polymerase (Takara).

\section{Isolation of flagellar filaments and monomeric flagellins}

Flagellar filaments were isolated in accordance with protocols described elsewhere [21]. Briefly, bacterial cells at mid exponential phase (OD600 of 1.0) were collected and vortexed intensively in distilled water. Cells were then removed by centrifugation at $8,000 \times \mathrm{g}$ for $5 \mathrm{~min}$. The cell free supernatants were then fractionated by ultracentrifugation. Pellets were suspended in a small amount of distilled water and stored at $-20{ }^{\circ} \mathrm{C}$ until use. For preparation of monomeric flagellins, the purified flagellar filaments were incubated in $6 \mathrm{M}$ urea solution and diluted with the medium used for Caco- 2 cell culture (described below).

\section{SDS-PAGE and staining}

Protein samples were prepared by mixing with an equal volume of $2 \times$ Laemmli buffer containing $5 \%$ betamercaptoethanol and boiled for $5 \mathrm{~min}$. Proteins were separated in 5-20\% polyacrylamide gradient c-PAGEL (ATTO) gels by SDS-PAGE. Protein bands were visualized by either staining with Quick-CBB PLUS (Wako) or periodic acid Schiff (PAS) staining. For PAS staining, gels were pretreated with $40 \%$ methanol and $7 \%$ acetic acid for $1 \mathrm{~h}$. After $1 \mathrm{~h}$ incubation with $7.5 \%$ acetic acid, gels were treated with $1 \%$ periodic acid for $1 \mathrm{~h}$ and washed intensively in $7.5 \%$ acetic acid. Glycoproteins were then stained with Schiff's reagent for $1 \mathrm{~h}$ and washed with $0.5 \%$ sodium bisulfite solution.

Matrix Assisted Laser Desorption/lonization time-of-flight mass spectrometry (MALDI-TOF MS)

Protein bands were enzymatically digested in-gel by treatment with porcine trypsin as described previously 
(Shevchenko et al. Anal. chem. 1996, 68:850-858). Briefly, gel pieces were washed with $50 \%$ acetonitrile to remove SDS, salt and CBB stain. Washed and dehydrated spots were then vacuum dried to remove solvent and rehydrated with trypsin $(8-10 \mathrm{ng} / \mu \mathrm{l})$ solution in $50 \mathrm{mM}$ ammonium bicarbonate $\mathrm{pH} 8.7$ and incubated $8-10 \mathrm{~h}$ at $37{ }^{\circ} \mathrm{C}$. Samples were analyzed using the Applied Biosystems 4700 proteomics analyzer with $\mathrm{TOF} / \mathrm{TOF}^{\mathrm{mm}}$ ion optics. MS data was acquired with a Nd: YAG laser with $200 \mathrm{~Hz}$ repetition rate, and up to 4000 shots were accumulated for each spectrum. MS data was acquired using the instrument default calibration, without applying internal or external calibration. These procedures were done by Genomine, Inc. (Korea).

\section{Flagella depolymerization assay}

Depolymerization of flagellar filaments were assessed by heating at $37-67^{\circ} \mathrm{C}$, incubation in $0.05-50 \mathrm{mM} \mathrm{HCl}$ solution at room temperature, or treatment with $5-5000 \mu \mathrm{g} / \mathrm{ml}$ of SDS at room temperature. After the treatments for $10 \mathrm{~min}, \mathrm{HCl}$ was neutralized by adding $0.5 \mathrm{M}$ of $\mathrm{NaHCO}_{3}$ solution for $50 \mathrm{mM}$ of $\mathrm{HCl}$ or $50 \mathrm{mM}$ of Tris- $\mathrm{Cl}(\mathrm{pH} 8.0)$ for lower $\mathrm{HCl}$ concentrations. The treated samples were mixed with equal volumes of $2 \times$ Native-PAGE sample buffer (30 \% glycerol, $1 \%$ bromophenol blue, $125 \mathrm{mM}$ Tris$\mathrm{HCl}, \mathrm{pH}$ 6.8). Samples were then loaded to c-PAGEL compact gels (ATTO) and electrophoresed using TrisGlycine buffer (BIO-RAD). After $30 \mathrm{~min}$ of electrophoresis (Native-PAGE), the gels were stained with CBB. As references, non-treated flagella mixed with Native-PAGE sample buffer (filament) and the protein mixed with Laemmli buffer containing $\beta$-melcaptoethanol (monomeric flagellin) were also prepared.

\section{Caco-2 cell-stimulation assay}

Caco-2 cells, originating from the human colon, were maintained in Dulbecco's modified Eagle Medium (DMEM, Wako) supplemented with $10 \%$ fetal bovine serum (BD), non-essential amino acids, glutamax (Thermo Fisher Scientific), and penicillin/streptomycin in $5 \% \mathrm{CO}_{2}$ at $37{ }^{\circ} \mathrm{C}$. Semi-confluent cultures of Caco-2 cells were collected by treatment with Tripsisn-EDTA solution (Life Technologies) and washed with the medium. The cells were then seeded to 96-well flat-bottom microplate wells (Thermo Fisher Scientific) at a concentration of $2 \times 10^{4}$ cells/well. Flagellin solution $(100 \mathrm{pmol} / \mathrm{ml})$ was added to each well and incubated for $4 \mathrm{~h}$. Culture supernatants were then collected and stored at $-20{ }^{\circ} \mathrm{C}$ until use. Human interleukin-8 (IL-8) was quantified with the OptEIA kit (BD) in accordance with the manufacturer's instructions. Assays were duplicated or triplicated and repeated three times at least. Tukey's multiple comparison test was performed and $\mathrm{P}$ values less than 0.05 were considered as statistically significant.

\section{Competing interests}

The authors declare that they have no competing interests.

\section{Authors' contributions}

AK involved in all experiments and prepared the manuscript. EM and KM contributed to isolation of flagella, Caco-2 cell culture, ELISA, and some preliminary experiments. KK, TI, SI, and SO contributed to prepare and review the manuscript. All authors read and approved the final manuscript.

\section{Acknowledgements}

We thank Evelyn Durmaz of North Carolina State University for grammatical correction and valuable comments. This work was supported by JSPS KAKENHI Grant Number 26850055

\section{Author details}

${ }^{1}$ Department of Applied Biology and Chemistry, Tokyo University of Agriculture, 1-1-1 Sakuragaoka, Setagaya, Tokyo 156-8502, Japan. ²Division of Biomedical Food Research, National Institute of Health Sciences, 1-18-1 Kamiyoga, Setagaya, Tokyo 158-8501, Japan. ${ }^{3}$ Department of Animal Science, Tokyo University of Agriculture, 1737 Funako, Atsugi, Kanagawa 243-0034, Japan.

Received: 7 September 2015 Accepted: 7 March 2016

Published online: 22 March 2016

\section{References}

1. Baele M, Devriese LA, Haesebrouck F. Lactobacillus agilis is an important component of the pigeon crop flora. J Appl Microbiol. 2001;91:488-91.

2. Stephenson DP, Moore RJ, Allison GE. Lactobacillus strain ecology and persistence within broiler chickens fed different diets: identification of persistent strains. Appl Environ Microbiol. 2010;76:6494-503.

3. Ngoc Lan P, Binh L, Benno Y. Impact of two probiotic Lactobacillus strains feeding on fecal lactobacilli and weight gains in chicken. J Gen Appl Microbiol. 2003;49:29-36

4. Al Jassim RAM. Lactobacillus ruminis is a predominant lactic acid producing bacterium in the caecum and rectum of the pig. Lett Appl Microbiol. 2003; 37:213-7.

5. Sharpe ME, Latham MJ, Garvie El, Zirngibl J, Kandler O. Two New Species of Lactobacillus Isolated from the Bovine Rumen, Lactobacillus ruminis sp.nov. and Lactobacillus vitulinus sp.nov. J Gen Microbiol. 1973;77:37-49.

6. Hayashi F, Smith KD, Ozinsky A, Hawn TR, Yi EC, Goodlett DR, Eng JK, Akira $\mathrm{S}$, Underhill DM, Aderem A. The innate immune response to bacterial flagellin is mediated by Toll-like receptor 5. Nature. 2001;410:1099-103.

7. Neville BA, Forde BM, Claesson MJ, Darby T, Coghlan A, Nally K, Ross RP, O'Toole PW. Characterization of pro-inflammatory flagellin proteins produced by lactobacillus ruminis and related motile lactobacilli. PLoS One. 2012;7:e40592

8. Korhonen JM, Sclivagnotis Y, Von Wright A. Characterization of dominant cultivable lactobacilli and their antibiotic resistance profiles from faecal samples of weaning piglets. J Appl Microbiol. 2007;103:2496-503.

9. Ocaña VS, Bru E, De Ruiz Holgado AA, Nader-Macias ME. Surface characteristics of lactobacilli isolated from human vagina. J Gen Appl Microbiol. 1999:45:203-12.

10. Stephenson DP, Moore RJ, Allison GE. Transformation of, and heterologous protein expression in, Lactobacillus agilis and Lactobacillus vaginalis isolates from the chicken gastrointestinal tract. Appl Environ Microbiol. 2011;77:220-8.

11. Wells J. Mucosal vaccination and therapy with genetically modified lactic acid bacteria. Annu Rev Food Sci Technol. 2011;2:423-45.

12. Schirm M, Kalmokoff M, Aubry A, Thibault P, Sandoz M, Logan SM. Flagellin from Listeria monocytogenes Is Glycosylated with $\beta$-O-Linked N Flagellin from Listeria monocytogenes Is Glycosylated with. Bacteriol. 2004;186:6721-7.

13. Logan SM. Flagellar glycosylation - a new component of the motility repertoire? Microbiology. 2006;152(Pt 5):1249-62.

14. Hayakawa J, Ishizuk M. Flagellar Glycosylation: Current Advances. Glycosylation. 2012;48352(InTech):127-52.

15. Verma A, Arora SK, Kuravi SK, Ramphal R. Roles of Specific Amino Acids in the N Terminus of Pseudomonas aeruginosa Flagellin and of Flagellin Glycosylation in the Innate Immune Response. Infect Immun. 2005;73:8237-46. 
16. Yoon S, Kurnasov O, Natarajan V, Hong M, Gudkov AV, Osterman AL, Wilson IA. Structural Basis of TLR5-Flagellin Recognition and Signaling. Science. 2012;335:859-64.

17. Smith KD, Andersen-Nissen E, Hayashi F, Strobe K, Bergman MA, Barrett SLR, Cookson BT, Aderem A. Toll-like receptor 5 recognizes a conserved site on flagellin required for protofilament formation and bacterial motility. Nat Immunol. 2003;4:1247-53.

18. Andersen-Nissen E, Smith KD, Strobe KL, Barrett SLR, Cookson BT, Logan SM, Aderem A. Evasion of Toll-like receptor 5 by flagellated bacteria. Proc Natl Acad Sci U S A. 2005;102:9247-52.

19. Kajikawa A, Ichikawa E, Igimi S. Development of a highly efficient proteinsecreting system in recombinant Lactobacillus casei. J Microbiol Biotechnol. 2010;20:375-82

20. Becavin C, Bouchier C, Lechat P, Archambaud C, Creno S, Gouin E, Wu Z, Kuhbacher A, Brisse S, Pucciarelli MG, Garcia-del Portillo F, Hain T, Portnoy DA, Chakraborty T, Lecuit M, Pizarro-Cerda J, Moszer I, Bierne H, Cossart P. Comparison of Widely Used Listeria monocytogenes Strains EGD, 10403S, and EGD-e Highlights Genomic Differences Underlying Variations in Pathogenicity. MBio. 2014;5.

21. Andersen-Nissen E, Smith KD, Bonneau R, Strong RK, Aderem A. A conserved surface on Toll-like receptor 5 recognizes bacterial flagellin. J Exp Med. 2007; 204:393-403.

\section{Submit your next manuscript to BioMed Central} and we will help you at every step:

- We accept pre-submission inquiries

- Our selector tool helps you to find the most relevant journal

- We provide round the clock customer support

- Convenient online submission

- Thorough peer review

- Inclusion in PubMed and all major indexing services

- Maximum visibility for your research

Submit your manuscript at www.biomedcentral.com/submit 